\title{
Zur Thermodynamik der Kristallgitter II.
}

Von H. Born und E. Brody in Göttingen.

Mit einer Abbildung. (Eingegangen am 26. August 1921.)

Einleitung. In einer unter gleichem Titel veröffentlichten Abhandlung ${ }^{1}$ ) hat der eine von uns ein Verfabren angegeben, die thermischen Eigenschaften der Kristalle aus der Gittertheorie abzuleiten. Dabei wurden die Zusammenhänge, die zwischen den verschiedenen thermischen Parametern (den Konstanten der Elastizität, Piezoelektrizität, thermisehen Ausdehnung, Pyroelektrizität und der Umkehreffekte) bestehen, aufgedeckt und Regeln angegeben, wie die Werte der Parameter ans den Atomkräften bei gegebener Gitterstruktur berechnet werden können. Zugleich wurde auch die Abbängigkeit aller Parameter von der Temperatur auf Grund der Quantentheorie entwickelt und das merkwürdige Ergebnis gewonnen, daß das früher von Grïneisen für die thermische Ansdehnung gefundene Gesetz nicht exalkt gültig sein soll. Nach Grüneisen ist der Koeffizient der thermischen Ausdehnung bei tiefen Temperaturen der spezifischen Wärme proportional, fällt also wie $T^{3}$ zu Null ab, während die Gittertheorie Proportionalität mit $T$ ergab. Dieses Resultat schien ${ }^{2}$ ) in Messungen über Pyroelektrizität von $\mathrm{Ackermann}{ }^{3}$ ) und älteren theoretischen Deutungen derselben durch.Boguslawskit) eine Stütze zu finden.

Wir haben nun den Versuch gemacbt, nach den angegebenen Regeln für Ionengitter vom Steinsalztypus mit Hilfe der Annahme elektrostatischer Kohäsion die thermische Ausdehnung wirklich zu berechnen. Dabei stellte sich herans, daß in der allgemeinen Theorie ein Fehler steckt, der den in der früheren Arbeit gefundenen Widerspruch gegen das Grüneisensche Gesetz versehuldet hat. Die dort aufgestellten Formein für die Temperaturabhängigkeit der Parameter sind also nicht richtig. Der Fehlschluß liegt in der Näherungsmethode, die bei der Auflösung der Gleichungen (49) angewandt wurde; bei dieser muß vorausgesetzt werden, daß die sämtlichen $3 s$ Wurzeln $\boldsymbol{\Omega}_{j}$ der Säkulargleichung von ( 49 a) einfach sind, und zwar für jeden Wert von $\tau=\frac{2 \pi}{\lambda}$, und sie sind es tatsächlich im allgemeinen auch, ansgenommen für $\tau=0$. Für diesen Wert ist aber bekanntlich $\boldsymbol{\Omega}^{0}=0$

1) M. Born, ZG. f. Phys. 7, 217, 1921. Im folgenden zitiert als Th. K. I.

2) M. Born, Phys. ZS. 23, 125, 1922.

3) W. Aekermann, Diss., Göttingen 1914; Ann. d. Phys. (4) 46, 197, 195.

4) S. Boguslawski, Phys. ZS. 15, 569, 805, 1914. 
eine dreifache Wurzel. Die folgenden Formeln sind dann für jedes von Null verschiedene $\tau$ allerdings richtig; aber schließlich wird bei der Anwendung des Debyeschen Näherungsverfahrens eine Entwicklung nach Potenzen von $\tau$ benutzt, und hier müssen die Formeln versagen, da sie gerade bei $\tau=0$ nicht gelten.

Wird der Fehler richtiggestellt, so führt die Gittertheorie im Bereiche tiefster Temperaturen anf das Grüneisensche Gesetz (Proportionalität mit $T^{3}$ ), und zwar nicht nur für die thermische Ansdehnung, sondern auch für die Pyroelektrizität, im Widerspruch mit den Messungen Ackermanns. Wio dieser Widerspruch zu beheben ist, können wir nicht sagen; es scheint uns jeđenfalls wünschenswert, daß die pyroelektrischen Messungen bei tiefen Temperaturen noch einmal mit größerer Genauigkeit wiederholt würden.

Den Hauptteil dieser Arbeit bildet die Durchrechnung der zweiatomigen, regulären Kristalle vom Steinsalztypus. Dabei gilt es vor allem der Entscheidung der Frage, ob die Annahme elektrostatischer Kohäsionskräfte ebenso wie zur Berechnung der elastischen Eigenschaften und der ultraroten Frequenzen auch zur Erklärung der thermischen Ausdehnung ausreicht. Da sich in früheren Berechnungen der Elastizitätskonstanten und der Reststrahlfrequenzen ein geringfügiger Rechenfehler gefunden hat, werden diese Rechnungen wiederbolt; es zeigt sich, daß nunmehr die Genauigkeit der Theorie ausreicht, um einen Schluß anf das Verhältnịs und Vorzeichen der Kräfte zu zichen, die zwischen gleichartigen und ungleichartigen Ionen umgekehrt proportional ungefähr zur zehnten Potenz der Entfernung wirken. Das Resultat ist [in Übereinstimmung mit dem früher bei Zinkblende ${ }^{1}$ ) gefundenen], daß diese Kräfte bei ungleichartigen Ionen Abstoßungen, bei gleichartigen aber Auziehungen sind. Dieses Ergebnis scheint uns für das Verständnis der Natur dieser Kräfte von Bedentung zu sein.

Für die thermische Ausdehnung erbält man im Bereiche tiefster Temperaturen' Proportionalität mit $T^{3}$, also mit der Atomwärme, wie es das Grüneisensche Gesetz verlangt; das Verhältnis beider Größen läßt sich aus den Atomkräften berechnen, und man erhält dafür leicht einen Ausdruck, von dem eine beträchtliche Genauigkeit erwartet: worden darf. Leider liegen keine Messungen des Ansdehnnngskoeffizienten bei Kristallen vom Steinsalatypus in diesem Temperatarbereich vor. Bei höheren Temperaturen läßt die Theorie, wie schon

1) M. Born a. E. Bormann, Verh. d. D. Phys. Ges. 21, 733, 1919; Ann. d. Phys. 62, 218, 1920. 
Grün eisen ${ }^{1}$ ) erkannt hat, keine Proportionalität zwischen Atomwärme und Aasdebnungskoeffizient erwarten. Im Grenzfall hoher Temperaturen (Bereich des Gesetzes von Dulong und Petit) werden beide Größen konstant, und man erhält für ihr Verhältnis mit Hilfe der von Debye entwickelten Näherangsmethode einen Ausdruck, der beträchtlich größer ist als der entsprechende Grenzwert beim absoluten Nullpankt. Doch ist nicht zu erwarten, daß diese Formel eine exakte Ubereinstimmung zwischen Rechnung und Beobachtung liefern wird. Bei der genannten Näherangsmethode werden nämlich die beiden Typen von Gitterschwingungen verschieden behandelt: Die langsamen (elastischen) werden nach dem Vorgange von Debye so berechnet, als wäre das Gitter ein Kontinuum; die Frequenzen werden daher der Wellenlänge nmgekehrt proportional. Die schnellen (ultraroten optischen) Schwingungen aber werden näherangsweise durch eine konstante, von der Wellenlänge unabhängige Grenzfrequenz dargestellt. Während dieses Verfahren bei der spezifischen Wärme für hohe Temperaturen unbedenklich ist, ist es für die thermische Ansdehnung zu roh; es zeigt sich, daß auf diese Weise die schnellen (ultraroten) Schwingungen zu viel Einfluß gewinnen, und dadurch fallen die berechneten Ausdehnungskoeffizienten etwas zu groB aus. Hier hätte eine Verfeinerung der Theorie einzusetzen.

Wir haben die numerischen Rechnungen zwar nicht mit großer Genauigkeit, aber in beträchtlicher Breite ausgefübrt, um eine Übersicht über die quantitativen Verhältnisse zu gewinnen, die qualitativ bercits von Grün eisen vollständig aufgeklärt worden sind. Im ganzen glauben wir in den gewonnenen Ergebnissen eine Bestätigung der Annahme elektrostatischer Kohäsionskräfte sehen zu dürfen.

§1. Berechnung der freien Energie. Wir knüpfen an die Formel (60) der zitierten Arbeit Th. K. I. an, durch welche die Dichte der freien Energie des Gitters $F$ dargestellt wird.

Für die immer wieder vorkommenden quantentheoretischen Funktionen führen wir folgende Abkürzungen ein:

$$
\begin{aligned}
& \mathbf{F}(x)=\ln \left(1-e^{-x}\right), \quad \mathbf{P}(x)=\frac{x}{e^{x}-1}, \\
& \mathbf{D}(x)=\frac{3}{x^{3}} \int_{0}^{x} y^{2} \mathbf{P}(y) d y=\frac{3}{x^{3}} \int_{0}^{x} \frac{y^{3}}{e^{y}-1} d y \\
& \mathbf{S}(x)=\mathbf{P}(x)-x \mathbf{P}^{\prime}(x)=\frac{x^{2} e^{x}}{\left(e^{x}-1\right)^{2}} .
\end{aligned}
$$

1) E. Grüneisen, 2. Conseil de Physique Bolvay 1913. Molekulartheorie der festen Körper. Siehe insbes. V, 8. 41. 
Statt $d \varphi_{1} d \varphi_{2} d \varphi_{3}$ schreiben wir einfach $d \varphi$. Dann wird

$$
F=U_{0}+U_{2}+\frac{k T}{(2 \pi)^{3} A} \int \sum_{j} \mathbf{F}\left(\frac{h v_{j}^{*}}{k T}\right) d \varphi .
$$

Dabei sind die $\boldsymbol{v}_{j}^{*}$ die Eigenfrequenzen des homogen deformierten Gitters, dessen potentielle Energie nach Th. K. I, (36) durch

$$
\Psi=N \mathcal{A}\left(U_{0}+U_{2}\right)+\frac{1}{4} \sum_{k k^{\prime}} \sum_{7 l^{\prime}} \sum_{x y}\left(\varphi_{k k^{\prime}}^{l-l^{\prime}}\right)_{x y}^{*} \mathfrak{v}_{k k^{\prime} x}^{l l^{\prime} x} \mathfrak{v}_{k k^{\prime} y}^{l l^{\prime}}
$$

gegeben ist, mit

$$
\left.\begin{array}{rl}
\left(\varphi_{k k^{\prime}}^{l}\right)_{x y}^{*} & =\left(\varphi_{k k^{\prime}}^{l}\right)_{x y}+\sum_{z}\left(\varphi_{k k^{\prime}}^{l}\right)_{x y z} \mathfrak{u}_{k k^{\prime} z}^{l} \\
& =\left(\varphi_{k k^{\prime}}^{l}\right)_{x y}+\sum_{z}\left(\varphi_{k k^{\prime}}^{l}\right)_{x y z}\left[\mathfrak{u}_{k z}-\mathfrak{H}_{k^{\prime} z}+\sum_{\bar{z}} u_{z \bar{z}} \bar{z}_{k k^{\prime}}^{l}\right]
\end{array}\right\}
$$

Wegen des in der Einleitung erläuterten singulären Verhaltens der Frequenzen bei $\tau=0$ läßt sich keine für alle $\tau$ geltende Darstellung für sie gewinnen. Wir beschränken uns daher von vornherein auf den Grenzfall unendlich langer Wellen, d. h. auf die Umgebung von $\tau=0$; man weiß aus der Theorie der spezifischen Wärmen, daß man so für manche Zwecke eine hinreichende Annäherung gewinnt. Bezeichnet man mit $j=1,2,3$ die drei langsamen (akustischen), mit $4,5, \ldots 3 p$ die schnellen (optischen) Eigenfrequenzen des $p$-atomigen Gitters, so gilt bekanntlich in erster Näherung:

$$
\left.\begin{array}{rl}
v_{j}^{*}=\frac{c_{j}^{*}}{2 \pi} \tau & (j=1,2,3) \\
\nu_{j}^{*}=v_{j}^{0 *} & (j=4,5, \ldots 3 p)
\end{array}\right\}
$$

und man erhält nach einfachen Umformungen

$$
F=U_{0}+U_{2}+\frac{k T}{\Delta}\left\{\sum_{j=1}^{3} \overline{\left[\mathbf{F}\left(\frac{\Theta_{j}^{*}}{T}\right)\right.}-\frac{1}{3} \overline{\left.\mathbf{D}\left(\frac{\Theta_{j}^{*}}{T}\right)\right]}+\sum_{j=4}^{3 p} \mathbf{F}\left(\frac{\Theta_{j}^{*}}{T}\right)\right\}
$$

dabei bedeutet der Strich Mittelbildung über die Einheitskugel und es ist

$$
\left.\begin{array}{ll}
\Theta_{j}^{*}=\frac{h v_{j}^{*}}{k}, & v_{j}^{*}=c_{j}^{*} \sqrt[3]{\frac{3}{4 \pi A}} \quad(j=1,2,3), \\
\Theta_{j}^{*}=\frac{h \nu_{j}^{*}}{k} & (j=4,5, \ldots 3 p) .
\end{array}\right\}
$$

Die $c_{j}^{*}$ sind die Schallgeschwindigkeiten, die $\grave{v}_{j}^{*}$ die Grenzfrequenzen des deformierten Gitters; sie sind aus den Koeffizienten (4) der potentiellen Energie zu ermitteln und werden, wie diese, lineare Funktionen der Deformationskomponenten $\mathfrak{a}_{k}, u_{x y}$. 
Der Gang der Rechnung ist folgender 1). Man bilde zunächst die Klammersymbole Th. K. I, (26) für das deformierte Gitter

$$
\left.\begin{array}{rl}
{\left[\begin{array}{c}
k k^{\prime} \\
x y
\end{array}\right]^{*}} & =\frac{1}{\Delta} S_{l}\left(\varphi_{k k^{\prime}}^{l}\right)_{x y}^{*}, \\
{\left[\begin{array}{c}
k \\
x y z
\end{array}\right]^{*}} & =\frac{1}{\Delta} \sum_{k^{\prime}} S_{l}\left(\varphi_{k k^{\prime}}^{l}\right)_{x y}^{*} z_{l k k^{\prime}}^{l}, \\
{[x y \bar{x} \bar{y}]^{*}} & =\frac{1}{2 \Delta} \sum_{k k^{\prime}} \boldsymbol{S}_{l}\left(\varphi_{k k^{\prime}}^{l}\right)_{x y}^{*} \bar{x}_{k k^{\prime}}^{l}, \overline{y_{k}} l k^{\prime}
\end{array}\right\}
$$

sodann durch Auflösen der linearen Gleichungen [die Th. K. I, (77) entsprechen]

$$
\sum_{k^{\prime}} \sum_{y}\left[\begin{array}{l}
k k^{\prime} \\
x y
\end{array}\right]^{*} \mathfrak{u}_{k^{\prime} y}=\mathfrak{H}_{k x}
$$

die Klammersymbole $\left\{\begin{array}{c}\left.k k^{k}\right\}^{\prime} \\ x y\end{array}\right\}^{*}$, definiert durch [Th. K. I, (88)]

$$
\mathfrak{u}_{k x}=\sum_{k^{\prime}} \sum_{y}\left\{\begin{array}{l}
k \cdot k^{\prime} y \\
x y
\end{array}\right\}^{*} \mathfrak{H}_{k^{\prime} y}
$$

und daraus die Elastizitätskonstanten des deformierten Gitters [Th. K. I, (86 c)]

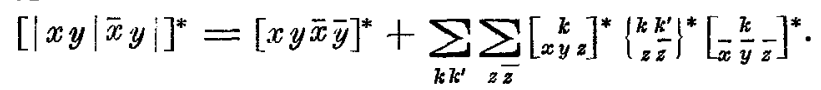

Alle diese Größen werden in erster Näherung lineare Funktionen von $\mathfrak{n}_{k x}, u_{x y}$.

Die Gleichungen für die Fortpflanzung elastischer Wellen lauten dann

$$
\rho c^{* 2} \mathfrak{u}_{x}-\sum_{\bar{x}} \mathfrak{u}_{\bar{x}} \sum_{y \bar{y}}[|x y| \bar{x} \bar{y} !]^{*} \xi_{y} \xi_{\bar{y}}=0
$$

und die Schwingungøgleichungen für die Grenzfrequenzen

$$
\left(2 \pi \bar{\nu}^{*}\right)^{2} m_{k} \mathfrak{u}_{k x}+\Delta \sum_{k^{\prime}} \sum_{y}\left[\begin{array}{l}
k k^{\prime} y^{\prime} \\
x y
\end{array} \mathfrak{u}_{k^{\prime} y}=0 .\right.
$$

Durch Nullsetzen der Determinanten dieser beiden Gleichungssysteme ergeben sich eine kubische Gleichung für das Quadrat der Schallgeschwindigkeit $c^{*}$ und eine Gleichung $3(p-1)^{\text {ten }}$ Grades für das Quadrat der Grenzfrequenz $i^{*}$. Ihre Wurzeln

$$
c_{1}^{*}, c_{2}^{*}, c_{3}^{*}, i_{4}^{*}, \ldots i_{3}^{*} p
$$

1) Ganz ähnliche Betrachtungen finden sich in Abhandlungen von M. J. M. v. Everdingen (Proefschrift, Utrecht 1914), H. A. Lorentz (Versl. Kon. Akad. 24, 671, 1915), Ornstein und Zernike (I, II, III, ebenda 24, 1561, 1689, 1916; 25, 396, 1916), J. Tresling (Proefschrift., Leiden 1919). Doch ist das Ziel dieser Arbeiten nicht die Zurückführung der Konstanten der thermischen Ausdehnung auf die Atomkräfte, sondern auf meBbare Abweichungen vom Hookeschen Gesetz. Daher ist die Art des Näherungsverfahrens auch durchans anders als in der vorliegenden Abhandlung. 
werden lineare Funktionen der $\mathfrak{u}_{k x}, u_{x y}$. So erhält man schließlich Ausdrücke der Form

$$
\Theta_{j}^{*}=\Theta_{j}\left(1-\sum_{k} \mathfrak{B}_{k}^{j} \mathfrak{u}_{k}-\sum_{x y} B_{x y}^{j} u_{x y}\right)
$$

wo die Komponenten der Vektoren $\mathfrak{B}_{k}^{j}$ und der Tensoren $B_{x y}^{j}$ für $j=1,2,3$ Funktionen der Wellenrichtung \& für $j=4,5, \ldots 3 p$ Konstante sind. Der Tensor ist symmetrisch

$$
B_{x y}^{j}=B_{y x}^{j}
$$

und von den $p$ Vektoren $\mathfrak{B}_{k}^{j}(k=1,2, \ldots p)$ sind nur $p-1$ unabbängig, da die Identität

gelten muß.

$$
\sum_{k} \mathfrak{B}_{k}^{j}=0
$$

Nun wird die freie Energie pro Volumeneinheit [Th. K. I, (66)]

$$
F=F_{0}-\sum_{k} \mathfrak{\Re}_{k}^{0} \mathfrak{u}_{k}-\sum_{x y} K_{i y}^{0} u_{x y}+U_{2},
$$

wo

$$
\left.\begin{array}{ll}
F_{0} & \left.=U_{0}+\frac{k T}{\Delta}\left\{\sum_{j=1}^{3} \overline{\left[\mathbf{F}\left(\frac{\Theta_{j}}{T}\right)\right.}-\frac{1}{3} \overline{\mathbf{D}\left(\frac{\Theta_{j}}{T}\right)}\right]+\sum_{j=4}^{3 p} \mathbf{F}\left(\frac{\Theta_{j}}{T}\right)\right\}, \\
\mathfrak{R}_{k}^{0} & =\quad \frac{k T}{\Delta}\left\{\sum_{j=1}^{3} \overline{\mathfrak{B}_{k}^{j} \mathbf{D}\left(\frac{\Theta_{j}}{T}\right)}+\sum_{j=4}^{3 p} \mathfrak{B}_{k}^{j} \mathbf{P}\left(\frac{\Theta_{j}}{T^{\prime}}\right)\right\}, \\
K_{x y}^{0} & =\quad \frac{k T}{\Delta}\left\{\sum_{j=1}^{3} \overline{B_{x y}^{j} \mathbf{D}\left(\frac{\Theta_{j}}{T}\right)}+\sum_{j=4}^{3 p} B_{x y}^{j} \mathbf{P}\left(\frac{\Theta_{j}}{T}\right)\right\} .
\end{array}\right\}
$$

Daraus folgt für die Energiedichte im unverzerrten Zustande:

$$
E_{0}=F_{0}-T \frac{d F_{0}}{d T}=U_{0}+\frac{k T}{\Delta}\left\{\sum_{j=1}^{3} \overline{\mathbf{D}\left(\frac{\Theta_{j}}{T}\right)}+\sum_{j=4}^{3 p} \mathbf{P}\left(\frac{\Theta_{j}}{T}\right)\right\} .
$$

Diese Formeln treten an die Stelle der unrichtigen Th. K.I. (61), $(62),(63)$.

Alle Schlüsse in dieser Arbeit aber, die nicht die Temperaturabhängigkeit der Parameter, sondern ibre formalen Zusammenhänge betreffen, bleiben ungeändert bestehen und sollen hier nicht wicderholt werden.

§2. Das Grüneisensche Gesetz. Die Grenzfälle tiefer und hoher Temperaturen. Wir beschräuken uns von jetzt an der Einfàcbheit halber auf reguläre Kristalle von zentrischer Symmetrie. Bei diesen sind die $K_{x y}^{0}$ direkt die thermischen Spannungen, and es gilt aus Symmetriegründen

$$
K_{x x}^{0}=K_{y y}^{0}=K_{z z}^{0}, \quad K_{y z}^{0}=K_{z x}^{0}=K_{x y}^{0}=0 .
$$


Der Koeffizient der linearen Ausdehnung ist

$$
\begin{aligned}
\alpha=\frac{x}{3} \frac{d K_{x x}^{0}}{d T}=\frac{\varkappa}{3} \cdot \frac{k}{\Delta}\left\{\sum_{j=1}^{3} \overline{B_{x x}^{j}\left[4 \mathbf{D}\left(\frac{\Theta_{j}}{T}\right)\right.}-3 \mathbf{P}\left(\frac{\Theta_{j}}{T}\right)\right] \\
\left.+\sum_{j=4}^{3 p} B_{x x}^{j} \mathbf{S}\left(\frac{\Theta_{j}}{T}\right)\right\},
\end{aligned}
$$

wobei $x$ den Modnl der kubischen Kompressibilität bedeutet.

Die Atomwärme bei fehlender Deformation ist

$$
C_{v}=V \frac{d E_{0}}{d T}=V \frac{k}{A}\left\{\sum_{j=1}^{3} \overline{\left[4 \mathbf{D}\left(\frac{\Theta_{j}}{T}\right)-3 \mathbf{P}\left(\frac{\Theta_{j}}{T}\right)\right]}+\sum_{j=4}^{3 p} \mathbf{S}\left(\frac{\Theta_{j}}{T}\right)\right\},
$$

wo das Atomvolumen

eingeführt ist.

$$
V=\frac{N \Delta}{p}(N=\text { Loschmidtsche Zahl pro Mol })
$$

Der Vergleich von (18) und (19) zeigt, daß $\alpha$ und $C_{v}$ nicht streng proportional sind, wie es das Grüneisensche Gesetz verlangt. Wohl aber gilt dies bei tiefen Temperaturen, im Bereich des Debyeschen $T^{3}$-Gesetzes. Denn für große $x$ verschwinden die Funktionen $\mathbf{P}(x)$ and $\mathbf{S}(x)$ exponentiell und es wird

Also erbält man

$$
\lim x^{3} \mathbf{D}(x)=\frac{\pi^{4}}{5}
$$

$$
\left.\begin{array}{rl}
\alpha & =\frac{x}{3} \cdot \frac{k}{d} \cdot \frac{4 \pi^{4}}{5} \sum_{j=1}^{3} \frac{\overline{B_{x}^{j}}}{\Theta_{j}^{3}} \cdot T^{3} \\
C_{v} & =V \cdot \frac{k}{d} \cdot \frac{4 \pi^{4}}{5} \sum_{j=1}^{3} \frac{\overline{1}}{\Theta_{j}^{3}} \cdot T^{3} \cdot
\end{array}\right\}
$$

Wir bilden nun die schon von $\mathrm{Mie}^{1}$ ) und $\mathrm{Grüneisen}{ }^{2}$ ) in ihren Untersuchungen über die Zustandsgleichnng der festen Körper eingeführte Größe

$$
\gamma=\frac{3 V \alpha}{x C_{v}}
$$

Dann zeigen die Formeln (21), daß diese bei sehr tiefen Temperaturen konstant ist, und zwar gleich

$$
\gamma_{0}=\lim _{T=0} \gamma=\frac{\sum_{j=1}^{3} \frac{\overline{B_{x x}^{j}}}{\Theta_{j}^{3}}}{\sum_{j=1}^{3} \frac{\overline{1}}{\Theta_{j}^{3}}} .
$$

1) G. Mie, Ann. d. Phys. 11, 657, 1903.

2) E. Grüneisen, ebenda 39, 257, 1912; 55, 371, 1918; 58, 758, 1919. 2. Conseil Solvay, Brüssel 1913. 
Das Grüneisensche Gesetz ist also für sehr tiefe Temperaturen exakt richtig.

Man kann nun den aus der Debyeschen Theorie der spezifischen Wärme bekannten Mittelwert $\bar{\Theta}$ durch

$$
\frac{3}{\overline{\bar{\Theta}^{3}}}=\sum_{j=1}^{3} \frac{1}{\Theta_{j}^{3}}
$$

einführen und näherungsweise die einzelnen $\Theta_{j}$ im Zähler von $\gamma_{0}$ durch $\bar{\Theta}$ ersetzen; dann wird

$$
\gamma_{0}=\frac{1}{3} \sum_{j=1}^{3} \overline{B_{x x}^{j}} \text {. }
$$

Im entgegengesetzten Grenzfalle hoher Temperaturen werden $\alpha$ und $C_{v}$ konstant, und zwar erhält man:

$$
\left.\begin{array}{rl}
\alpha & =\frac{x}{3} \cdot \frac{k}{\Delta}\left\{\sum_{j=1}^{3} \overline{B_{x x}^{j}}+\sum_{j=4}^{3} B_{x x}^{j}\right\}, \\
C_{v} & =V \frac{k}{\Delta} 3 p=3 N k=3 R .
\end{array}\right\}
$$

Wir bilden nun wieder die durch (22) definierte Größe $\gamma$ und erbalten

$$
\gamma_{\infty}=\lim _{x=\infty} \gamma=\frac{1}{3 p}\left\{\sum_{j=1}^{3} \overline{B_{x x}^{j}}+\sum_{j=4}^{3 p} B_{x x}^{j}\right\} .
$$

Um den Utbergang von $T=0$ bis $T=\infty$ übersichtlich darzustellen, kann man in den allgemeinen Formeln (18) und (19) nach $\bar{D}$ ebyes Vorgang die $\Theta_{j}$ der akustischen Anteile durch $\Theta$ ersetzen; führt man dann die Funktionen

ein, so erhält man

$$
\Psi_{j}\left(T^{\prime}\right)=\frac{\mathbf{S}\left(\frac{\Theta_{j}}{T}\right)}{4 \mathbf{D}\left(\frac{\bar{\Theta}}{T}\right)-3 \mathbf{P}\left(\frac{\bar{\Theta}}{T}\right)}
$$

$$
\gamma=\frac{\sum_{j=1}^{3} \overline{B_{x x}^{j}}+\sum_{j=4}^{3 p} B_{x x}^{j} \Psi_{j}^{j}(T)}{3+\sum_{j=4}^{3 p} \Psi_{j}(T)},
$$

und da $\Psi(0)=0, \Psi(\infty)=1$ ist, sieht man sofort, daß die Grenzfälle $\gamma_{0}, \gamma_{\infty}$ durch (25) und (27) richtig wiedergegeben werden.

Wir wollen uns auf die Betrachtung von $\gamma_{0}$ und $\gamma_{\infty}$ beschränken. Der Unterschied beider Größen rührt von dem mit wachsender Temperatur bemerkbar werdenden Einflu $\beta$ der altraroten Eigenschwingungen her. Hierzu ist aber folgende Bemerkung zu machen: 
Die Formeln (17) und damit auch (27) beruhen auf der Annahme, daß die schnellen (optischen) Eigenfrequenzen $v_{j}^{*}$ durch die konstanten Grenzfrequenzen $i_{j}^{*}(5)$ ersetzt werden dürfen. Aber das ist keineswegs unter allen Umständen erlaubt; man sieht das besonders deutlich, wenn man ein zweiatomiges, reguläres Gitter betrachtet, dessen beide Atome hinsichtlich ihrer Masse und ihrer wechselseitigen Kraftwirkungen nahezu gleich sind. Dann verhält sich das Gitter offenbar fast wie ein einatomiges; die schnellen Eigenfrequenzen $\nu_{j}^{*}$ werden also auch nicht annähernd von der Wellenlänge unabhängig sein. Die freie Energie ist dann sinngemäß nur durch drei Debyesche Funktionen darzustellen. Zwischen diesem Grenzfall und dem umgekehrten, wo die Atome sehr verschieden sind, wird es alle Übergänge geben; man ist aber heute noch nicht in der Lage, diese durch ein rationelles Näherungsverfahren zu behandeln. Wir werden sehen, daß bei der thermischen Ausdehnung für hohe Temperaturen die Anteile der langsamen und schuellen Frequenzen wesentlich verschieden sind und das Resultat davon abhängt, ob man die schnellen Schwingungen durch eine konstante Grenzfrequenz ersetzen darf.

Eine vollständige Theorie hätte auf die strenge Formel (2) zurückzugehen und die darin vorkommende Funktion $\mathbf{F}(x)$ für kleine Werte von $x$ (entsprechend großen Werten von $T$ ) durch $\ln x$ zu ersetzen; dann wird

wo $\overline{\nu^{*}}$ den durch

$$
F=U_{0}+U_{2}+\frac{p k T}{\Delta} \ln \frac{h \cdot *}{k T}
$$

$$
\ln \overline{\nu^{*}}=\frac{1}{(2 x)^{3} p} \int \ln \left(\nu_{1}^{*} \nu_{2}^{*} \ldots \nu_{3 p}^{*}\right) d \varphi
$$

definierten Mittelwert bedeutet. Nun kann man, wie schon O. Stern ${ }^{1}$ ) bemerkt hat, das Produkt aller Frequenzen auf die Determinante der Schwingungsgleichungen zarückführen. Doch scheint vorlänfig kein Weg bekannt zu sein, um von hier aus zu übersichtlichen Ergebnissen zu gelangen; wir müssen die Darehführung dieses Gedankens der Zukunft überlassen.

Hier wollen wir uns daranf beschränken, die beiden Ausdruicke $\gamma_{0}$ und $\gamma_{\infty}$ nach (25) und (27) zu berechnen, von denen der erste bei tiefen Temperaturen mit großer Annäberung gilt, der zweite bei hohen wahrscheinlich zu groß ausfallen wird. Die Prüfung der Theorie kann dann nur darin bestehen, $\mathrm{da} B$ die aus den Beobachtungen bei hohen Temperataren berechneten Werte von $\gamma$ zwisehen die berechneten Zahlen $\gamma_{0}$ und $\gamma_{\infty}$ fallen.

1) O. Stern, Ann. d. Phys. (4) 51, 237, 1916. 
Bei der Berechnung der in (25) und (27) vorkommenden Summen kann man den schon erwähnten Gedanken von Stern benutzen, der es ermöglicht, mit rationalen Operationen auszukommen. Nach (13) ist nämlich

also wird

$$
B_{x y}^{j}=-\frac{\partial \ln \Theta_{j}^{*}}{\partial u_{x y}} ;
$$

$$
\begin{aligned}
& \sum_{j=1}^{3} B_{x y}^{j}=-3 \frac{\partial \ln \bar{\Theta}^{*}}{\partial u_{x y}}, \\
& \sum_{j=4}^{3 p} B_{x y}^{j}=-3(p-1) \frac{\partial \ln \bar{\Theta} 0}{\partial u_{x y}}
\end{aligned}
$$

wo $\Theta^{*}=\frac{h \overline{\nu^{*}}}{k}, \Theta^{0}=\frac{h \overline{\nu_{0}}}{k}$ Mittelwerte bedeuten, die so definiert sind:

$$
\begin{aligned}
\Theta^{*} 3 & =\left(\frac{h \overline{\nu *}}{k}\right)^{3}=\Theta_{1}^{*} \Theta_{2}^{*} \Theta_{3}^{*}=\left(\frac{h}{k}\right)^{3} \nu_{1}^{*} \nu_{2}^{*} v_{3}^{*}, \\
\Theta_{0} 3(p-1) & =\left(\frac{h \bar{\nu})}{k}\right)^{3(p-1)}=\Theta_{4}^{*} \ldots \Theta_{3 p}^{*}=\left(\frac{h}{h}\right)^{3(p-1)} \bar{v}_{4}^{*} \ldots \tilde{\nu}_{3 p}^{*} .
\end{aligned}
$$

Diese lassen sich aber rational aus den Koeffizienten der Schwingungsgleichungen (11) und (12) herstellen; dann setzt man

so wird

$$
\begin{aligned}
& \Pi^{*}=\operatorname{Det} .\left(\sum_{y \bar{y}}[|x y| \bar{x} \bar{y} \mid]^{*} \varepsilon_{y} \xi_{\bar{y}}^{-}\right), \\
& \Pi^{0}=\operatorname{Det} .\left(\begin{array}{c}
{\left[\begin{array}{l}
k k^{\prime} \\
x y
\end{array}\right]^{*}} \\
k, k^{*}=1,2, \ldots(p-1)
\end{array}\right),
\end{aligned}
$$

also

$$
\begin{aligned}
& \left(\nu_{1}^{*} \nu_{2}^{*} \nu_{3}^{*}\right)^{2} \text { proportional } \Pi^{*} \text {, } \\
& \left(\stackrel{\nu}{*}_{4}^{*} i_{b}^{*} \ldots \tilde{\nu}_{3 p}^{*}\right)^{2} \quad, \quad \Pi^{0} \text {, }
\end{aligned}
$$

$$
\begin{aligned}
3 \ln \overline{\Theta^{*}} & =1 / 2 \ln \Pi^{*}+\text { konst., } \\
3(p-1) \ln \overline{\Theta^{0}} & =1 / 2 \ln \Pi^{0}+\text { konst. }
\end{aligned}
$$

Nun sind $\Pi^{*}$ und $\Pi^{0}$ Funktionen der $\mathfrak{1}_{k}, u_{x y}$; entwickelt man sie nach diesen und setzt

so erhält man

$$
\left.\begin{array}{l}
\Pi^{*}=\Pi_{0}^{*}\left(1-\sum_{k} \mathfrak{B}_{k}^{*} \mathfrak{u}_{k}-\sum_{x y} B_{x y}^{*} u_{x y}\right), \\
\Pi^{0}=\Pi_{0}^{0}\left(1-\sum_{k} \mathfrak{B}_{k}^{0} \mathfrak{u}_{k}-\sum_{x y} B_{x y}^{0} u_{x y}\right),
\end{array}\right\}
$$

$$
\left.\begin{array}{l}
\sum_{j=1}^{3} B_{x y}^{j}=1 / 2 B_{x y}^{*}, \\
\sum_{j=4}^{3 p} B_{x y}^{j}=1 / 2 B_{x y}^{0} .
\end{array}\right\}
$$


Hier hängt $B_{x y}^{*}$ noch von $\&$ ab, während $B_{x y}^{0}$ konstant ist. Man bekommt schließlich nach (25) and (27)

$$
\left.\begin{array}{l}
\gamma_{0}=\frac{1}{6} \overline{B_{x x}^{*}}, \\
\gamma_{\infty}=\frac{1}{6 p}\left(\overline{B_{x x}^{*}}+B_{x x}^{\prime}\right) .
\end{array}\right\}
$$

Die Mittelbilduug in $\gamma_{0}$ ist nicht ganz elementar ausführbar, weil es sich um gebrochen rationale Funktionen von $\varepsilon_{x}$, bandelt; wir werden diese Schwierigkeit umgehen, indem wir uns mit einer Annäberung begnügen.

§3. Zuräckführung der physikalischen Parameter auf Gittersummen. Wir beschränken uns jetzt auf zweiatomige, regu- läre, zentrische Gitter vom Steinsalztypus $(p=2)$.

Zunächst sind die Klammersymbole (8) zu bilden; diese sind sämtlich Null außer den folgenden ${ }^{1}$ ):

$$
A^{*}=[x x x x]^{*}, \quad B^{*}=[x x y y]^{*}, \quad D_{k k^{\prime}}^{*}=\left[\begin{array}{l}
k k^{\prime} \\
x x
\end{array}\right]^{*} ;
$$

alle durch zyklische Vertauschung der $x, y, z$ aus diesen hervorgehenden Klammergrößen sind einander gleich. Ferner ist

$$
D_{12}^{*}=D_{21}^{*}=-D_{11}^{*}=-D_{22}^{*}=D^{*}=\left[\begin{array}{c}
12 \\
x x
\end{array}\right]^{*} \text {. }
$$

Bei rein thermischer Deformation gilt aus Symmetriegründen

$$
u_{k}=0, \quad u_{x x}=u_{y y}=u_{z z}=u, \quad u_{y z}=u_{z x}=u_{x y}=0 .
$$

Daher werden die $A^{*}, B^{*}, D^{*}$ lineare Funktionen ron $u$; wir setzen

$$
A^{*}=A+u A^{\prime}, \quad B^{*}=B+u B^{\prime}, \quad D^{*}=D+u D^{\prime} .
$$

Hier sind $A, B, D$ die Konstanten des undeformierten Gitters, für die man die Ausdrücke erhält [Th. K. I, (26)]:

$$
\begin{aligned}
& A=\frac{1}{2 \Delta} \sum_{k k^{\prime}} \int_{l} Q_{k k^{\prime}}^{l}\left(x_{k k^{\prime}}^{l}\right)^{4}, \\
& B=\frac{1}{2 \Delta} \sum_{k k^{\prime}} S_{l} Q_{k k^{\prime}}^{l}\left(y_{k k^{\prime}}^{l}\right)^{2}\left(z_{k k^{\prime}}^{l}\right)^{2}, \\
& D=\frac{1}{\Delta} S_{l}\left\{P_{12}^{l}+Q_{12}^{l}\left(x_{12}^{l}\right)^{2}\right\} .
\end{aligned}
$$

Die kubische Kompressibilität $x$ bängt mit $A$ und $B$ so zusammen:

$$
\frac{3}{x}=A+2 B=\frac{1}{2 \Delta} \sum_{k k^{\prime}} \sum_{l} Q_{k k^{\prime}}^{l}\left(r_{k k^{\prime}}^{l}\right)^{4}
$$

1) Die Bezeichnung ist in Übereinstimmung mit der früher gebrauchten (s. M. Born, Phys. ZS. 19, 539, 1918; Ann. d. Phys. 61, 87, 1919; M. Born und E. Bormann, Ann. d. Phys. 62, 218, 1920). 
sie läßt sich ferner durch die potentielle Energie aller Zellen des Gitterg auf eine (gleich der doppelten Energie des Gitters pro Zelle)

so ausdrücken:

$$
\varphi_{0}=\sum_{k k^{\prime}} S_{i}^{\prime} \varphi_{k k k^{\prime}}^{l}
$$

$$
\frac{1}{x}=\frac{1}{18 \delta} \frac{d^{2} \varphi_{0}}{d \delta^{2}} \text {. }
$$

Für die Konstanten des deformierten Gitters erhält man nun aus (8), indem man (4) einsetzt und die Symmetrieeigenschaften berücksichtigt:

$$
\begin{aligned}
& A^{\prime}=3 A+2 B+\frac{1}{2 A} \sum_{k k^{\prime}} S_{l} R_{k k^{\prime}}^{l}\left(r_{k k^{\prime}}^{l}\right)^{2}\left(x_{k k^{\prime}}^{l}\right)^{4} \\
& B^{\prime}=A+4 B+\frac{1}{2 A} \sum_{k k^{\prime}} S_{l} R_{k k^{\prime}}^{l}\left(r_{k k^{\prime}}^{l}\right)^{2}\left(y_{k k^{\prime}}^{l}\right)^{2}\left(z_{k k^{\prime}}^{l}\right)^{2} \\
& D^{\prime}=5 D-\frac{5}{A} S_{l} P_{12}^{l}+\frac{1}{3 A} S_{l} R_{12}^{\prime}\left(r_{12}^{l}\right)^{4}
\end{aligned}
$$

Statt $B^{\prime}$ kann man auch die bequemere Größe

$$
A^{\prime}+2 B^{\prime}=5(A+2 B)+\frac{1}{6 \Delta} \sum_{k k^{\prime}} \int_{l} R_{k k^{\prime}}^{l}\left(r_{k k^{\prime}}^{l}\right)^{6}
$$

benutzen. Ferner erkennt man leicht die Gültigkeit der Relation ${ }^{1}$ )

$$
D^{\prime}=\frac{1}{\delta^{2}} \frac{d \Delta D}{d \delta}
$$

Wegen der zentrischen Symmetrie sind die $A^{*}, B^{*}$ direkt gleich den Elastizitätskonstanten (10); in der Voigtschen Bezeichnung wird man zu schreiben haben:

$$
\left.\begin{array}{l}
c_{11}=A, \quad c_{12}=c_{44}=B, \\
c_{11}^{*}=A^{*}=A+u A^{\prime}, \quad c_{12}^{*}=c_{44}^{*}=B^{*}=B+u B^{\prime} .
\end{array}\right\}
$$

Jetzt kann man ohne weiteres die Schwingungsgleichungen (11) und (12) oder die zugehörigen Determinanten (30) bilden. Man erhält direkt also nach $(31)$

$$
\Pi^{0}=D^{* 3}=\left(D+u D^{\prime}\right)^{3}
$$

$$
B_{x x}^{0}=-\frac{D^{\prime}}{D} \text {. }
$$

Ebenso leicht läßt sich die Determinante der elastischen Schwingungen $\Pi^{*}$ hinschreiben; doch kommt man dann bei der Mittelbildung über die Einheitskugel zu den schon erwähnten Schwierigkeiten. Um diese zu umgehen, wollen wir die vereinfachende Annahme machen, daß der (undeformierte) Kristall elastisch nahezu isotrop ist,

1) Für $A^{\prime}, B^{\prime}$ gelten keine analogen Relationen; das hängt damit zusammen, daß die Ausdrücke (38) für $A, B$ nicht für beliebige Gitterkonstante $\delta$, sondern nur für das Gleichgewicht gelten, so daß man sie nicht nach $\delta$ differentiieren darf. 
d.h. daB mit genügender Annäherung $c_{11}=3 c_{12}, A=3 B$ gesetzt werden kann. Das ist bei Steinsalz nahezu erfüllt. Sodann erhält man

wo

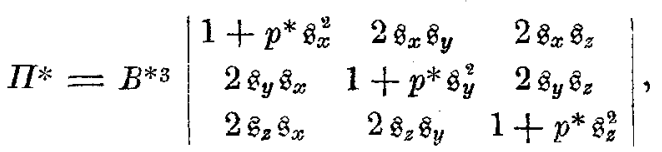

$$
p^{*}==\frac{A^{*}}{B^{*}}-1=2+u 3\left(\frac{A^{\prime}}{A}-\frac{B^{\prime}}{B}\right)+\cdots
$$

gesetzt ist. Eutwickelt man nun $\Pi^{*}$ nach $u$, so wird

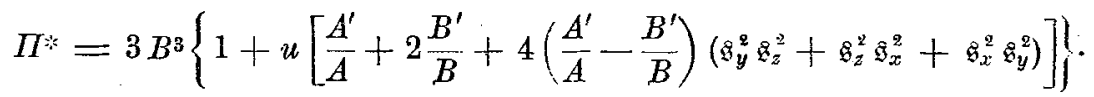

Nach (31) bat man somit

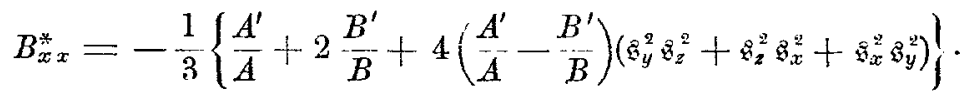

Durch Mittelbildang folgt daraus

$$
\bar{B}_{x x}^{*}=-\frac{1}{3}\left\{\begin{array}{l}
A^{\prime} \\
A
\end{array}+\frac{2 B^{\prime}}{B}+\frac{4}{5}\left(\frac{A^{\prime}}{A}-\frac{B^{\prime}}{B}\right)\right\}=-\frac{1}{5}\left(3 \frac{A^{\prime}}{A}+2 \frac{B^{\prime}}{B}\right) .
$$

Setzt man nun (46) und (48) in (33) ein, so erhält man (mit $p=2$ ):

$$
\left.\begin{array}{rl}
\gamma_{0} & =-\frac{1}{30}\left(3 \frac{A^{\prime}}{A}+2 \frac{B^{\prime}}{B}\right), \\
\gamma_{\infty} & =-\frac{1}{60}\left(3 \frac{A^{\prime}}{A}+2 \frac{B^{\prime}}{B}+5 \frac{D^{\prime}}{D}\right) .
\end{array}\right\}
$$

Wie schon gesagt, muß man erwarten, daß der empirische Wert von $\gamma$ zwischen diesen beiden Grenzwerten liegt.

8. Berechnung der Gittersummen für das Steinsalzgitter. Für die Gitter vom Typus des Steinsilzes wählt man am besten als Zelle das Rhomboeder ${ }^{1)}$ nit den Kanten

$\left.\begin{array}{c}\mathfrak{a}_{1}=r_{0}\left(\mathfrak{i}_{2}+\mathfrak{i}_{3}\right), \quad \mathfrak{a}_{2}=r_{0}\left(\mathfrak{i}_{3}+\mathfrak{i}_{1}\right), \\ \mathfrak{a}_{3}=r_{0}\left(\mathfrak{i}_{1}+\dot{i}_{2}\right) ;\end{array}\right\}$

dabei sind $\mathfrak{i}_{1}, \mathfrak{i}_{2}, \mathfrak{t}_{3}$ die Einheitsvektoren parallel zu den Würfelkanten und $r_{0}$ ist der Abstand eines Paares benachbarter Na- und Cl-Ionen (s. Figur). Das Zellenvolumen ist dann

$$
\Delta=\delta^{3}=2 r_{0}^{3} \text {. }
$$

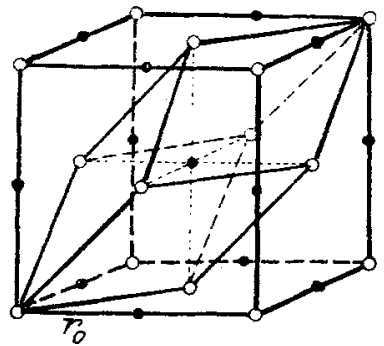

1) Siehe M. Born, Ann. d. Phys. 61, 87, 1919. Dort ist die Bezeichnung etwas anders; während hier $\delta$ die dritte Wurzel aus dem Zellenvolumen $d$ bedeutet, ist dort $\delta$ gleich dem Abstand zweier gleicher Ionen längs der Würfelkante, also in der hier gebrauchten Bezeichnung gleich $2 r_{0}$. 
Die Zelle entbält je ein Na- und ein Cl-Ion, deren Lage man so wählen kann

$$
\mathfrak{r}_{1}=0, \quad \mathfrak{r}_{2}=r_{0}\left(\grave{t}_{1}+\dot{t}_{2}+\dot{\mathfrak{t}}_{3}\right) \text {. }
$$

Es wird demnach

$$
\left.\begin{array}{l}
\mathfrak{r}_{11}^{l}=\mathfrak{r}_{22}^{l}=r_{0}\left\{\mathfrak{i}_{1}\left(l_{2}+l_{3}\right)+\mathfrak{t}_{2}\left(l_{3}+l_{1}\right)+\mathfrak{t}_{3}\left(l_{1}+l_{2}\right)\right\}, \\
\mathfrak{r}_{21}^{l}=-\mathfrak{r}_{12}^{l}=r_{0}\left\{\dot{t}_{1}\left(1+l_{2}+l_{3}\right)+\mathfrak{t}_{2}\left(1+l_{3}+l_{1}\right)+\mathfrak{t}_{3}\left(1+l_{1}+l_{2}\right)\right\}
\end{array}\right\}
$$

Das elementare Potential entwickeln wir in eine Reihe nach fallenden Potenzen der Entfernung:

Dann erhält man

$$
\varphi_{k k^{\prime}}(r)=\sum_{p=1}^{\infty} \frac{b_{k k^{\prime}}^{(p)}}{r^{p}} \quad\left(k, k^{\prime}=1,2\right) .
$$

$$
\left.\begin{array}{ll}
\varphi_{k k^{\prime}}^{l}=\sum_{p=1}^{\infty} \frac{b_{k k^{\prime}}^{(p)}}{\left(r_{k k^{\prime}}^{l}\right)^{p}}, & P_{k k^{\prime}}^{l}=-\sum_{p=1}^{\infty} \frac{p b_{k k^{\prime}}^{(p)}}{\left(r_{k k^{\prime}}^{l}\right)^{p+2}}, \\
Q_{k k^{\prime}}^{l}=\sum_{p=1}^{\infty} \frac{p(p+2) b_{k k^{\prime}}^{(p)}}{\left(r_{k k^{\prime}}^{l}\right)^{p+4}}, & R_{k k^{\prime}}^{l}=-\sum_{p=1}^{\infty} \frac{p(p+2)(p+4) b_{k k^{\prime}}^{p}}{\left(r_{k k^{\prime}}^{l}\right)^{p+6}} .
\end{array}\right\}
$$

Man setze nun

ferner

$$
b_{12}^{(p)}=b_{p}, \quad \frac{b_{11}^{(p)}+b_{22}^{(p)}}{2 b_{12}^{(p)}}=\beta_{p},
$$

$$
\begin{aligned}
& S_{0}^{\prime}(p)=S_{l}\left[\left(1+l_{2}+l_{3}\right)^{2}+\left(1+l_{0}+l_{1}\right)^{2}+\left(1+l_{1}+l_{2}\right)^{2}\right]^{-\frac{p}{2}}, \\
& S_{0}^{\prime \prime}(p)=S_{l}^{\prime}\left[\left(l_{2}+l_{3}\right)^{2}+\left(l_{3}+l_{1}\right)^{2}+\left(l_{1}+l_{2}\right)^{2}\right]^{-\frac{p}{2}}, \\
& S_{1}^{\prime}(p)=3 S_{l}\left[\left(1+l_{2}+l_{3}\right)^{2}+\cdots\right]^{-\frac{p+4}{2}}\left(1+l_{2}+l_{3}\right)^{4}, \\
& S_{1}^{\prime \prime}(p)=3 S_{l}\left[\left(i_{2}+l_{3}\right)^{2}+\cdots\right]^{-\frac{p+4}{2}}\left(l_{2}+l_{3}\right)^{4} \\
& \text { und } \quad \begin{array}{c}
S_{0}(p)=S_{0}^{\prime}(p)+\beta_{p} S_{0}^{\prime \prime}(p), \\
S_{1}(p)=S_{1}^{\prime}(p)+\beta_{p} S_{1}^{\prime \prime}(p) ;
\end{array}
\end{aligned}
$$

dann wird nach (38) und (40)

$$
\left.\begin{array}{rl}
\varphi_{0} & =2 \sum_{p=1}^{\infty} \frac{1}{r_{0}^{p}} b_{p} S_{0}(p), \\
A & =\frac{1}{3} \sum_{p=1}^{\infty} \frac{1}{r_{0}^{p}} b_{p} p(p+2) S_{1}(p), \\
A+2 B & =\frac{1}{3 \Delta} \sum_{p=1}^{\infty} \frac{1}{r_{0}^{p}} b_{p} p(p+2) S_{0}(p), \\
B & =\frac{1}{6 \Delta} \sum_{p=1}^{\infty} \frac{1}{r_{0}^{p}} b_{p} p(p+2)\left\{S_{0}(p)-S_{1}(p)\right\} \\
D & =\frac{1}{3 \Delta} \sum_{p=1}^{\infty} \frac{1}{r_{0}^{p+2}} b_{p} p(p-1) S_{0}^{\prime}(p+2) .
\end{array}\right\}
$$


Zwischen $r_{0}$ und den Konstanten $b_{p}, \beta_{p}$ muß die Gleichgewichtsbedingung

$$
\frac{d \varphi_{0}}{d r_{0}}=-\frac{2}{r_{0}} \sum_{p=1}^{\infty} \frac{1}{r_{0}^{p}} b_{p} p S_{0}(p)=0
$$

bestehen. Sodann erhält man weiter aus (42), (43):

$$
\left.\begin{array}{rl}
A^{\prime} & =3 A+2 B-\frac{1}{3 \Delta} \sum_{p=1}^{\infty} \frac{1}{r_{0}^{p}} b_{p} p(p+2)(p+4) S_{1}(p), \\
A^{\prime}+2 B^{\prime} & =5(A+2 B)-\frac{1}{3 \Delta} \sum_{p=1}^{\infty} \frac{1}{r_{0}^{p}} b_{p} p(p+2)(p+4) S_{0}(p), \\
D^{\prime} & =5 D-\frac{1}{3 \Delta} \sum_{p=1}^{\infty} \frac{1}{r_{0}^{p+2}} b_{p} p\{(p+2)(p+4)-15\} S_{0}^{\prime}(p+2),
\end{array}\right\}
$$

oder mit Rücksicht auf (59):

$$
\begin{aligned}
& A^{\prime}=-\frac{1}{3 \Delta} \sum_{p=1}^{\infty} \frac{1}{r_{0}^{p}} b_{p} p(p+2)\left\{(p+2) S_{1}(p)-S_{0}(p)\right\} \\
& B^{\prime}=-\frac{1}{6 \Delta} \sum_{p=1}^{\infty} \frac{1}{r_{0}^{p}} b_{p} p(p+2)\left\{p S_{0}(p)-(p+2) S_{1}(p)\right\} \\
& D^{\prime}=-\frac{1}{3 \Delta} \sum_{p=1}^{\infty} \frac{1}{r_{0}^{p}} b_{p} p(p+2)(p-1) S_{0}^{\prime}(p+2) .
\end{aligned}
$$

Wir spezialisieren jetzt das Kraftgesetz in derselben Weise, wie es $\mathrm{Mie}^{1}$ ) und Grüneisen ${ }^{2}$ ) getan haben, indem wir nur zwei Glieder der Reihe (54) beibehalten, mil den Exponenten $p=m$ und $p=n>m$. Das erste soll eine Anziehung, das zweite eine Abstoßung bedeuten; wir setzen also

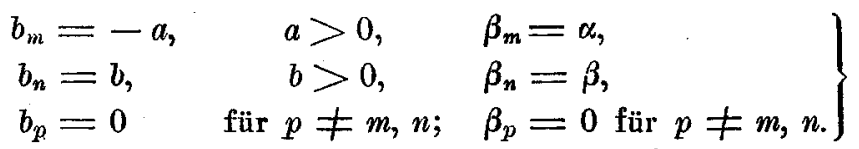

Dann kann man roch $b$ mit Hilfe von (60) eliminieren und erhält nach einfacher Rechnung:

$$
\left.\begin{array}{rl}
\varphi_{0} & =-2 \frac{a m}{r_{0}^{m}} S_{0}(m)\left(\frac{1}{m}-\frac{1}{n}\right), \\
A & =\frac{1}{3 \Delta} \frac{a m}{r_{0}^{m}} S_{0}(m) f_{1}(n, m), \\
A+2 B & =\frac{1}{3 \Delta} \frac{a m}{r_{0}^{m}} S_{0}(m)(n-m), \\
B & =\frac{1}{6 \Delta} \frac{a m}{r_{0}^{m}} S_{0}(m) f_{2}(n, m), \\
D & =\frac{1}{3 \Delta} \frac{a m}{r_{0}^{m+2}} S_{0}(m) f_{3}(n, m) ;
\end{array}\right\}
$$

1) G. Mie, Ann. d. Phys. (4) 11, 657, 1903.

2) E. Grüneisen, Ann. d. Phys. (4) 26, 211, 393, 1908; 39, 257, 1912; Verb. d. D. phys. Ges. 13, 836, 1911; 14, 322, 1912. 
dabei ist gesetzt:

$$
\left.\begin{array}{l}
f_{1}(n, m)=(n+2) \frac{S_{1}(n)}{S_{0}(n)}-(m+2) \frac{S_{1}(m)}{S_{0}(m)}, \\
f_{2}(n, m)=(n-m)-f_{1}(n, m), \\
f_{3}(n, m)=(n-1) \frac{S_{0}^{\prime}(n+2)}{S_{0}(n)}-(m-1) \frac{S_{0}^{\prime}(m+2)}{S_{0}(m)} \cdot
\end{array}\right\}
$$

Ans $n>m$ folgt $\varphi_{0}<0, A>0, A+2 B>0, D>0$; das Vorzeichen von $B$ ist nicht ohne weiteres anzugeben.

Die Formeln (65) liefern die Gitterenergie und die elastischen Konstanten $c_{11}=A, c_{12}=c_{44}=B$. Zwischen der Kompressibilität $x$, gegeben durch

$$
\frac{1}{x}=\frac{1}{3}(A+2 B)=\frac{1}{9 \Lambda} \frac{a m}{r_{0}^{m}} S_{0}(m)(n-m),
$$

und der Gitterenergie $\varphi_{0}$ besteht die einfache Beziehung

oder

$$
x \varphi_{0}=-18 \Delta \frac{1}{m n},
$$

$$
U_{0}=\frac{\Phi_{0}}{V_{0}}=\frac{\varphi_{0}}{2 A}=\frac{9}{m n} \cdot \frac{1}{x} ;
$$

hier bedeatet $V_{0}$ das Volumen, $\Phi_{0}$ die Energie des Gitters im Gleichgewicht, $U_{0}$ seine Energiedichte. Diese Relation findet sich für einatomige Körper in etwas anderer Form scbon bei Grüneisen 1). Weiter erhält man aus (62)

$$
\begin{aligned}
& A^{\prime}=-\frac{1}{3 \Delta} \frac{a m}{r_{0}^{m}} S_{0}(m) f_{1}^{\prime}(n, m), \\
& B^{\prime}=-\frac{1}{6 \Delta} \frac{a m}{r_{0}^{m}} S_{0}(m) f_{2}^{\prime}(n, m), \\
& D^{\prime}=-\frac{1}{3 A} \frac{a m}{r_{0}^{m+2}} S_{0}(m) f_{3}^{\prime}(n, m) ;
\end{aligned}
$$

dabei ist gesetzt:

$$
\left.\begin{array}{l}
f_{1}^{\prime}(n, m)=(n+2)\left[(n+2) \frac{S_{1}(n)}{S_{0}(n)}-1\right]-(m+2)\left[(m+2) \frac{S_{1}(m)}{S_{0}(m)}-1\right] \\
f_{2}^{\prime}(n, m)=(n-m)(n+m+1)-f_{1}^{\prime}(n, m), \\
f_{3}^{\prime}(n, m)=(n+2)(n-1) \frac{S_{0}^{\prime}(n+2)}{S_{0}(n)}-(m+2)(m-1) \frac{S_{0}^{\prime}(m+2)}{S_{0}(m)} \cdot
\end{array}\right\}
$$

1) A. a. O.Anm. 2, S. 341. Siehe insbes. Aun. d. Phys. 39, 257, 1912, Nr. 12, Formel (41). Dort ist $\boldsymbol{\Phi}_{0}$ mit der Sublimationswärme beim absoluten Nullpunkt $\left(\varrho_{13}\right)_{0}$ identifiziert; ferner sind die hier mit $m, n$ bezeichneten Exponenten dort $3 m, 3 n$ genannt. 
Nunmebr wird die Konstante der thermischen Ausdehnung (49):

$$
\begin{aligned}
\gamma_{0} & =\frac{1}{30}\left(3 \frac{f_{1}^{\prime}}{f_{1}}+2 \frac{f_{2}^{\prime}}{f_{2}}\right) . \\
\gamma_{\infty} & =\frac{1}{60}\left(3 \frac{f_{1}^{\prime}}{f_{1}}+2 \frac{f_{2}^{\prime}}{f_{2}}+5 \frac{f_{3}^{\prime}}{f^{\prime}}\right) .
\end{aligned}
$$

Diese Formeln sind in ihrer Struktur ganz analog den von Mie und Grüneisen für einatomige Körper aufgestellten Gesetzen.

Nimmt man an, daß $n$ sehr groß gegen $m$, also auch gegen 1 ist, so folgt ans (65) und (69) näherungsweise

also

$$
\frac{f_{1}^{\prime}}{f_{1}}=\frac{f_{2}^{\prime}}{f_{2}}=\frac{f_{3}^{\prime}}{f_{3}}=n+2 \text {, }
$$

$$
\gamma_{0}=\gamma_{\infty}=\frac{n+2}{6} \text {. }
$$

Mit diesem Wert hat Mie in seiner ersten Arbeit gerechnet, und Grüneisen hat ihn ebenfalls diskutiert.

Im allgemeinen hängt $\gamma$ von beiden Exponenten $m$ und $n$ ab, außerdem von der Konstanten $\beta$, welche das Verhältuis der Kräfte zwischen gleichartigen und ungleichartigen Ionen, bezogen auf gleichen Abstand, bedeutet.

§5. Elektrostatische Kohäsion. Wir machen jetzt die Annahme, daß die Kohäsionskräfte elektrostatischer Natur sind, daß also $m=1$ ist. Für diesen Fall ist die Berechnung der elastischen Konstanten und der Reststrahl-Wellenlänge bereits durchgeführt worden ${ }^{1}$ ), und wir können die früher mitgeteilten Resultate hier benutzen. Doch soll wegen eines Rechenfehlers in der zitierten Arbeit das Wesentliche bier in verbesserter Form wiederholt werden.

Für $m=1$ lassen sich die Gittersummen (57), (58) nicht direkt summieren, sondern müssen nach den Methoden von Madelung ${ }^{2}$ ) oder Ewald s) berechnet werden. Man hat offenbar

$$
a=e^{2}, \quad \alpha=-1
$$

zu setzen, wo $e$ die Ionenladung ist; dann wird

$$
S_{0}(1)=S_{0}^{\prime}(1)-S_{0}^{\prime \prime}(1), \quad S_{1}(1)=S_{1}^{\prime}(1)-S_{1}^{\prime \prime}(1),
$$

und durch die ganzzahligen Substitationen

$$
\left.\begin{array}{ll}
l_{1}^{\prime}=1+l_{2}+l_{3} & l_{1}^{\prime \prime}=l_{2}+l_{3} \\
l_{2}^{\prime}=1+l_{3}+l_{1} & l_{2}^{\prime \prime}=l_{3}+l_{1} \\
l_{3}^{\prime}=1+l_{1}+l_{2} & l_{3}^{\prime \prime}=l_{1}+l_{2}
\end{array}\right\}
$$

1) M. Born, Ann. d. Phys. 61, 87, 1919. Die entsprechenden Rechnungen über die Zinkblende (M. Born u. E. Bormann, Ann. d. Phys. 52, 218, 1920) enthalten einen analogen Rechenfehler und sollen demnächst wiederholt werden.

2) E: Madelung, Phys. ZS. 19, 524, 1918.

3) P. P. Ewald, Ann. d. Phys. 64, 253, 1921.

Zeitschrift flur Physik. Bd. XI. 
verwandeln diese Summen sich in

$$
\left.\begin{array}{l}
S_{0}(1)=-S_{l}^{\prime} \frac{(-1)^{l_{1}+l_{2}+l_{3}}}{\sqrt{l_{1}^{2}+l_{2}^{2}+l_{3}^{2}}}, \\
S_{1}(1)=-3 S_{l} \frac{(-1)^{l_{1}+l_{2}+l_{3}} l_{1}^{4}}{\left(\sqrt{l_{1}^{2}+l_{2}^{2}+l_{3}^{2}}\right)^{5}} \cdot
\end{array}\right\}
$$

Die erste Summe ist das elektrostatische Potential aller Gitterpnnkte außer dem Nullpunkt in diesem und hat den Wert ${ }^{1}$ )

$$
S_{0}(1)=1,747 \text {. }
$$

Ferner findet man leicht ${ }^{2}$ )

wo

$$
S_{1}(1)=1 / 3 S_{0}(1)-C,
$$

$$
\begin{aligned}
& C=\int_{i}(-1)^{l_{1}+l_{2}+l_{3}} l_{1}^{q}\left[\frac{\partial}{\partial x^{2}} \frac{1}{\sqrt{\left(x-l_{1}\right)^{2}+l_{2}^{2}+l_{3}^{2}}}\right]_{x=0} \\
& =2 \sum_{p=1}^{\infty}(-1)^{p} \cdot\left(\frac{\partial^{2} \Phi}{\partial x^{2}} x^{2}\right)_{x=p}
\end{aligned}
$$

gesetzt ist; dabei bedentet $\Phi$ das Potential der Netzebene $x=0$ in einem Aufpunkte $x, 0,0$. Diese Größe $C$ ist früher berechnet worden zu

daher erhält man

$$
C=-2,644
$$

$$
S_{1}(1)=3,226 \text {. }
$$

Das in $f_{8}$ und $f_{3}^{\prime}$ vorkommende Glied $(m-1) S_{0}^{\prime}(m+2)$, dessen erster Faktor für $m=1$ verschwindet, rührt von der Kraft her, die das Gitter der positiven Ionen auf das der negativen ausübt. Diese darf bekanntlich nicht aus dem gewöhnlichen, der Laplaceschen Differentialgleichung genügenden Potential abgeleitet werden, sondern aus einer Funktion $\varphi$, die der Gleichung

$$
\frac{\partial^{2} \varphi}{\partial x^{2}}+\frac{\partial^{2} \varphi}{\partial y^{2}}+\frac{\partial^{2} \varphi}{\partial z^{2}}=\frac{4 \pi e}{\Delta}
$$

genügt. Daher erbält man die Kraft pro Zelle

$$
-e \frac{\partial^{2} \varphi}{\partial x^{2}}=-\frac{4 \pi e^{2}}{3 \Delta}
$$

das ist der elektrodynamische Teil der Größe $\Delta D$. Der Vergleicb mit (64), (65) zeigt, daß man formal

zu setzen hat.

$$
(m-1) S_{0}^{\prime}(m+2)=2 \pi
$$

1) P. P. Ewald, Ann. d. Phys. 69, 253, 1921.

2) In der zitierten Arbeit (Anm. 1, S. 343) ist $S_{1}(1)$ einfach gleich $-C$ gesetzt [s. dort Anhang $(f),(g)$ ]. Darin besteht der oben erwähnte Rechenfehler. 
Für die Abstoßungskraft ist $n$ etwa gleich 9; eine Tabelle der Summen (57) findet sich in der zitierten Arbeit ${ }^{1}$ ), wobei allerdings die Bezeichnung etwas anders ist.

Mittels der Substitution (73) kann man schreiben:

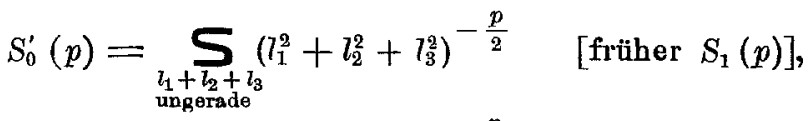

$$
\begin{aligned}
& S_{0}^{\prime \prime}(p)=\underbrace{\prime}_{\substack{l_{1}+l_{2}+l_{3} \\
\text { gerade }}}\left(l_{1}^{9}+l_{2}^{2}+l_{3}^{2}\right)^{-\frac{p}{2}} \quad\left[\text { früher } S_{2}(p)\right], \\
& S_{1}^{\prime}(p)=\underset{\substack{l_{1}+l_{2}+l_{3} \\
\text { ungerade }}}{3}\left(l_{1}^{2}+l_{2}^{2}+l_{3}^{2}\right)^{-\frac{p+4}{2}} l_{1}^{4} \quad\left[\text { früher } 3 C_{1}(p+4)\right] \\
& S_{1}^{\prime \prime}(p)=\underset{\substack{l_{1}+l_{2}+l_{3} \\
\text { gerade }}}{3}\left(l_{1}^{2}+l_{2}^{2}+l_{3}^{2}\right)^{-\frac{p+4}{2}} l_{1}^{4} \quad\left[\text { früher } 3 C_{2}(p+4)\right] .
\end{aligned}
$$

Die frïher mitgeteilte Tabelle nimmt nun folgende Gestalt an:

Tabelle 1.

\begin{tabular}{c|c|c|c|c|c}
\hline \hline$p$ & $S_{0}^{\prime}(p)$ & $S_{0}^{\prime \prime}(p)$ & $S_{0}^{\prime}(p+2)$ & $S_{1}^{\prime}(p)$ & $S_{1}^{\prime \prime}(p)$ \\
\hline 7 & 6,231 & 1,149 & 6,065 & 6,084 & 0,5739 \\
8 & 6,144 & 0,785 & 6,036 & 6,045 & 0,3942 \\
9 & 6,065 & 0,544 & 6,020 & 6,021 & 0,2730 \\
10 & 6,036 & 0,381 & 6,011 & 6,012 & 0,1908
\end{tabular}

Man hat nun zunächst den Abstoßungsexponenten aus der Gleichung (66) zu bestimmen, die für $m=1$ so lautet:

$$
n-1=\frac{18}{1,747} \cdot \frac{r_{0}^{4}}{e^{2}} \cdot \frac{1}{x} \text {. }
$$

Hier ist $r_{0}$ in bekannter Weise aus der Dichte $\varrho$ und den Atomgewichten $\mu_{1}, \mu_{2}$ zu bestimmen; man erhält mit $e=4,774.10^{-10} \mathrm{ESE}$ :

also

$$
\frac{e^{2}}{r_{0}^{4}}=2,947 \cdot 10^{13}\left(\frac{\varrho}{\mu_{1}+\mu_{2}}\right)^{4 / 3},
$$

$$
n=1+3,496 \cdot 10^{-13} \frac{1}{x}\left(\frac{\mu_{1}+\mu_{2}}{\varrho}\right)^{4 / 3} .
$$

Die folgende Tabelle gibt $n$ für 6 Na- und K-Halogenide. Die Kompressibilitäten sind, wie früher, einer Arbeit von Richards und Jones ${ }^{2}$ ) entnommen; nur bei $\mathrm{KCl}$ ist der etwas größere Wert der neuen Messung von Madelung und $\mathrm{Fuchs}{ }^{3}$ ) benutzt (bei $\mathrm{NaCl}$ stimmen

1) 1. c. Anm. 1, S. 343.

2) Th. W. Richards u. Gr. Jones, Journ. Amer. Chem. Soe. 31, 158, 1909.

3) E. Madelung u. R. Fuchs, Ann. d. Phys. (4) 65, 289, 1921. 
Tabelle 2.

\begin{tabular}{|c|c|c|c|c|c|}
\hline & $\mu_{1}$ & $\mu_{2}$ & $\varrho_{\text {beob. }}$ & $x_{\text {beob. }}$ & $n$ \\
\hline $\begin{array}{l}\mathrm{Na} \mathrm{Cl} . \\
\mathrm{Na} \mathrm{Br} \\
\mathrm{Na} J\end{array}$ & $\begin{array}{l}23,0 \\
23,0 \\
23,0\end{array}$ & $\begin{array}{r}35,5 \\
79,9 \\
126,9\end{array}$ & $\begin{array}{l}2,17 \\
3,01 \\
\mathbf{3}, \mathbf{5 5}\end{array}$ & $\begin{array}{l}4,13.10-12 \\
5,1 \\
6,9\end{array}$ & $\begin{array}{l}7,84 \\
8,61 \\
8,45\end{array}$ \\
\hline 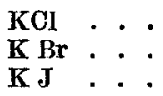 & $\begin{array}{l}39,1 \\
39,1 \\
39,1\end{array}$ & $\begin{array}{r}35,5 \\
79,9 \\
126,9\end{array}$ & $\begin{array}{l}1,98 \\
2,70 \\
3,07\end{array}$ & $\begin{array}{l}5,62 \\
6,2 \\
8,6\end{array}$ & $\begin{array}{l}8,86 \\
9,78 \\
9,31\end{array}$ \\
\hline
\end{tabular}

die beiden Messungen genau überein). Die Werte von $n$ liegen sämtlich zwischen 7 and 10; man kann daher die zugehörigen Werte der Gittersummen aus Tabelle 1 durch (graphische) Interpolation entnehmen und erhält damit die Tabelle 3 .

Tabelle 3.

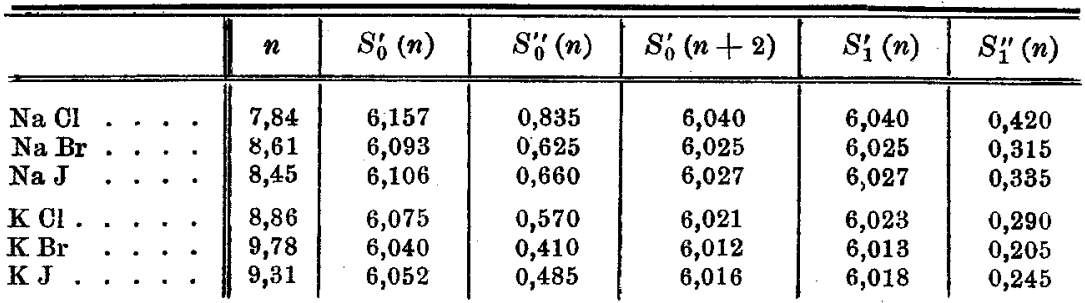

Nunmehr kann man die Funktionen (65) und (69) für jede einzelne Substanz tabulieren. Die Rechnung wurde mit der Genauigkeit des Rechenschiebers ausgeführt.

Tabelle 4.

\begin{tabular}{|c|c|c|c|c|c|c|c|c|c|}
\hline$f_{1}$ & & $\boldsymbol{\beta}=$ & $-1,5$ & $-1,0$ & $-0,5$ & 0 & 0,5 & 1,0 & 1,5 \\
\hline $\begin{array}{l}\mathrm{Na} \mathrm{OI} \\
\mathrm{Na} \mathrm{Br} \\
\mathrm{Na} \mathrm{J}\end{array}$ & $\dot{.}$ & 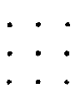 & $\begin{array}{l}5,28 \\
5,88 \\
5,74\end{array}$ & $\begin{array}{l}4,83 \\
5,56 \\
5,36\end{array}$ & $\begin{array}{l}4,45 \\
5,25 \\
5,05\end{array}$ & $\begin{array}{l}4,10 \\
4,96 \\
4,76\end{array}$ & $\begin{array}{l}3,81 \\
4,69 \\
4,46\end{array}$ & $\begin{array}{l}3,54 \\
4,47 \\
4,29\end{array}$ & $\begin{array}{l}3,30 \\
4,26 \\
4,07\end{array}$ \\
\hline $\begin{array}{l}\mathbf{K} \mathrm{Ol} \\
\mathbf{K} \mathbf{B r} \\
\mathbf{K} \mathbf{J}\end{array}$ & $\dot{.}$ & $\begin{array}{l}. \\
. \\
.\end{array}$ & $\begin{array}{l}6,08 \\
6,85 \\
6,46\end{array}$ & $\begin{array}{l}5,76 \\
6,59 \\
6,16\end{array}$ & $\begin{array}{l}5,47 \\
6,37 \\
5,94\end{array}$ & $\begin{array}{l}5,22 \\
6,17 \\
5,69\end{array}$ & $\begin{array}{l}4,99 \\
5,96 \\
5,47\end{array}$ & $\begin{array}{l}4,75 \\
5,85 \\
5,28\end{array}$ & $\begin{array}{l}4,56 \\
\mathbf{5 , 6 5} \\
\mathbf{5 , 2 1}\end{array}$ \\
\hline
\end{tabular}

Tabelle 5 .

\begin{tabular}{|c|c|c|c|c|c|c|c|c|}
\hline$f_{\mathbf{2}}$ & $\boldsymbol{\beta}=$ & $-1,5$ & $-1,0$ & $-0,5$ & 0 & 0,5 & 1,0 & 1,5 \\
\hline $\begin{array}{l}\mathrm{Na} \mathrm{Cl} \\
\mathrm{Na} \mathrm{Br} \\
\mathrm{Na} J\end{array}$ & 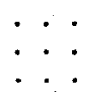 & $\begin{array}{l}1,56 \\
1,73 \\
1,71\end{array}$ & $\begin{array}{l}2,01 \\
2,05 \\
2,09\end{array}$ & $\begin{array}{l}2,39 \\
2,36 \\
2,40\end{array}$ & $\begin{array}{l}2,74 \\
2,65 \\
2,69\end{array}$ & $\begin{array}{l}3,03 \\
2,92 \\
2,99\end{array}$ & $\begin{array}{l}3,30 \\
3,14 \\
3,16\end{array}$ & $\begin{array}{l}3,54 \\
3,35 \\
3,38\end{array}$ \\
\hline $\begin{array}{l}\mathbf{K} \mathbf{O l} \\
\mathbf{K} \mathbf{B r} \\
\mathbf{K} \mathbf{J}\end{array}$ & 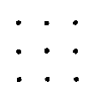 & $\begin{array}{l}1,78 \\
1,93 \\
1,85\end{array}$ & $\begin{array}{l}2,10 \\
2,19 \\
2,15\end{array}$ & $\begin{array}{l}2,39 \\
2,41 \\
2,37\end{array}$ & $\begin{array}{l}2,64 \\
2,61 \\
2,62\end{array}$ & $\begin{array}{l}2,87 \\
2,82 \\
2,84\end{array}$ & $\begin{array}{l}3,11 \\
2,93 \\
3,03\end{array}$ & $\begin{array}{l}3,30 \\
3,13 \\
3,10\end{array}$ \\
\hline
\end{tabular}


Tabelle 6.

\begin{tabular}{|c|c|c|c|c|c|c|c|c|}
\hline$f_{3}$ & $\beta=$ & $-1,5$ & $-1,0$ & $-0,5$ & 0 & 0,5 & 1,0 & 1,5 \\
\hline $\begin{array}{l}\mathrm{NaOl} \\
\mathrm{Na} \mathrm{Br} \\
\mathrm{NaJ} .\end{array}$ & & $\begin{array}{l}4,82 \\
5,31 \\
5,18\end{array}$ & $\begin{array}{l}4,17 \\
4,80 \\
4,64\end{array}$ & $\begin{array}{l}3,60 \\
4,35 \\
4,17\end{array}$ & $\begin{array}{l}3,11 \\
3.94 \\
3,75\end{array}$ & $\begin{array}{l}2,75 \\
3,56 \\
3,37\end{array}$ & $\begin{array}{l}2,31 \\
3,24 \\
3,04\end{array}$ & $\begin{array}{l}1,97 \\
2,93 \\
2,72\end{array}$ \\
\hline $\begin{array}{l}\mathrm{K} \mathbf{~} \mathbf{l} . \\
\mathrm{K} \mathbf{B r} \\
\mathbf{K} J\end{array}$ & & $\begin{array}{l}5,48 \\
6,13 \\
5,79\end{array}$ & $\begin{array}{l}5,01 \\
5,77 \\
5,38\end{array}$ & $\begin{array}{l}4,59 \\
5,44 \\
5,01\end{array}$ & $\begin{array}{l}4,20 \\
5,14 \\
4,66\end{array}$ & $\begin{array}{l}3,86 \\
4,85 \\
4,35\end{array}$ & $\begin{array}{l}3,53 \\
4,59 \\
4,05\end{array}$ & $\begin{array}{l}3,23 \\
4,32 \\
3,78\end{array}$ \\
\hline
\end{tabular}

Tabelle 7.

\begin{tabular}{|c|c|c|c|c|c|c|c|c|}
\hline$f_{i}^{\prime}$ & $\beta=$ & $-1,5$ & $-1,0$ & $-0,5$ & 0 & 0,5 & 1,0 & 1,5 \\
\hline $\begin{array}{l}\mathrm{NaCl} \\
\mathrm{Na} \mathrm{Br} \\
\mathrm{Na} \mathrm{J}\end{array}$ & $\cdots$ & $\begin{array}{l}83,0 \\
97,2 \\
93,6\end{array}$ & $\begin{array}{l}78,5 \\
\mathbf{9 3 , 5} \\
\mathbf{8 9 , 7}\end{array}$ & $\begin{array}{l}74,8 \\
90,3 \\
86,5\end{array}$ & $\begin{array}{l}71,4 \\
87,2 \\
83,6\end{array}$ & $\begin{array}{l}68,5 \\
84,4 \\
80,4\end{array}$ & $\begin{array}{l}65,9 \\
82,0 \\
78,6\end{array}$ & $\begin{array}{l}63,5 \\
79,9 \\
76,4\end{array}$ \\
\hline $\begin{array}{l}\mathbf{K} \mathbf{C l} . \\
\mathbf{K} \mathbf{B r} \\
\mathbf{K} \boldsymbol{J} .\end{array}$ & & $\begin{array}{l}101,6 \\
120,4 \\
110,8\end{array}$ & $\begin{array}{r}98,2 \\
117,5 \\
107,4\end{array}$ & $\begin{array}{r}95,2 \\
114,9 \\
104,8\end{array}$ & $\begin{array}{r}92,4 \\
112,5 \\
102,3\end{array}$ & $\begin{array}{r}89,7 \\
110,0 \\
99,6\end{array}$ & $\begin{array}{r}87,2 \\
108,5 \\
97,4\end{array}$ & $\begin{array}{r}84,9 \\
106,3 \\
96,6\end{array}$ \\
\hline
\end{tabular}

Tabelle 8.

\begin{tabular}{|c|c|c|c|c|c|c|c|c|}
\hline$f_{z}^{\prime}$ & $\beta=$ & $-1,5$ & $-1,0$ & $-0,5$ & 0 & 0,5 & 1,0 & 1,5 \\
\hline $\begin{array}{l}\mathrm{Na} B r \\
\text { Na J }\end{array}$ & & $\begin{array}{l}-15,7 \\
-16,4 \\
-15,6\end{array}$ & $\begin{array}{l}-11,2 \\
-12,7 \\
-11,7\end{array}$ & $\begin{array}{l}7,5 \\
-\quad 9,5 \\
-\quad 8,5\end{array}$ & $\begin{array}{l}-4,1 \\
-6,4 \\
-5,6\end{array}$ & $\begin{array}{l}-1,2 \\
-3,6 \\
-2,4\end{array}$ & $\begin{array}{l}+1,4 \\
-1,2 \\
-0,6\end{array}$ & $\begin{array}{r}+3,8 \\
+0,9 \\
+1,6\end{array}$ \\
\hline $\begin{array}{l}\mathrm{Ol} \\
\mathrm{Br} \\
\mathrm{J}\end{array}$ & & $\begin{array}{l}-16,2 \\
-17,0 \\
-16,8\end{array}$ & $\begin{array}{r}-12,8 \\
-14,1 \\
-13,4\end{array}$ & $\begin{array}{r}9,8 \\
-11,5 \\
-10,8\end{array}$ & $\begin{array}{l}-7,0 \\
-9,1 \\
-8,3\end{array}$ & $\begin{array}{l}-4,3 \\
-6,6 \\
-5,6\end{array}$ & $\begin{array}{l}-1,8 \\
-5,1 \\
-3,4\end{array}$ & $\begin{array}{r}+0,5 \\
-2,9 \\
-2,6\end{array}$ \\
\hline
\end{tabular}

Tabelle 9.

\begin{tabular}{|c|c|c|c|c|c|c|c|c|}
\hline$f_{3}^{\prime}$ & $\beta=$ & $-1,5$ & $-1,0$ & $-0,5$ & 0 & 0,5 & 1,0 & 1,5 \\
\hline $\begin{array}{l}\mathrm{Na} \mathrm{Ol} \\
\mathrm{Na} \mathrm{Br} \\
\mathrm{Na} \mathrm{J}\end{array}$ & & $\begin{array}{l}72,1 \\
83,8 \\
81,0\end{array}$ & $\begin{array}{l}65,7 \\
78,3 \\
75,2\end{array}$ & $\begin{array}{l}60,0 \\
73,6 \\
70,5\end{array}$ & $\begin{array}{l}55,2 \\
69,2 \\
66,1\end{array}$ & $\begin{array}{l}51,7 \\
65,2 \\
62,1\end{array}$ & $\begin{array}{l}47,4 \\
61,8 \\
58,6\end{array}$ & $\begin{array}{l}44,0 \\
58,5 \\
55,2\end{array}$ \\
\hline $\begin{array}{l}\mathrm{KCl} . \\
\mathrm{K} \mathbf{B r} \\
\mathrm{K} \mathbf{J}\end{array}$ & & $\begin{array}{r}87,7 \\
103,8 \\
95,3\end{array}$ & $\begin{array}{l}82,7 \\
99,4 \\
90.8\end{array}$ & $\begin{array}{l}78,1 \\
95,5 \\
86,7\end{array}$ & $\begin{array}{l}73,8 \\
92,1 \\
82,7\end{array}$ & $\begin{array}{l}70,2 \\
88,7 \\
79,2\end{array}$ & $\begin{array}{l}66,6 \\
85,6 \\
75,8\end{array}$ & $\begin{array}{l}63,3 \\
82,5 \\
72,7\end{array}$ \\
\hline
\end{tabular}

Endlich erhält man für die Grenzwerte der Größe $\dot{\gamma}$ nach (70) and $\left(70^{\prime}\right)$ : 
Tabelle 10.

\begin{tabular}{c|c||c|c|c|c|c|c|c}
\hline \hline$\gamma_{0}$ & $\beta=$ & $-1,5$ & $-1,0$ & $-0,5$ & 0 & 0,5 & 1,0 & 1,5 \\
\hline Na Ol . . . & 0,90 & 1,26 & 1,47 & 1,64 & 1,77 & 1,89 & 2,01 \\
Na Br... & 1,02 & 1,27 & 1,45 & 1,60 & 1,72 & 1,81 & 1,89 \\
Na J... & 1,02 & 1,30 & 1,48 & 1,62 & 1,75 & 1,82 & 1,91 \\
K Cl . . . & 1,06 & 1,30 & 1,47 & 1,59 & 1,70 & 1.80 & 1,87 \\
K Br... & 1,17 & 1,36 & 1,49 & 1,59 & 1,69 & 1,74 & 1,82 \\
K J . . . & 1,11 & 1,33 & 1,46 & 1,59 & 1,69 & 1,77 & 1,80
\end{tabular}

Tabelle 11

\begin{tabular}{|c|c|c|c|c|c|c|c|c|}
\hline$y_{\infty}$ & $\beta=$ & $-1,5$ & $-1,0$ & $-0,5$ & 0 & 0,5 & 1,0 & 1,5 \\
\hline $\begin{array}{l}\mathrm{Na} \mathrm{Cl} \\
\mathrm{Na} \mathrm{Br} \\
\mathrm{Na} J\end{array}$ & . & $\begin{array}{l}1,70 \\
1,82 \\
1,81\end{array}$ & $\begin{array}{l}1,94 \\
2,00 \\
2,00\end{array}$ & $\begin{array}{l}2,12 \\
2,14 \\
2,15\end{array}$ & $\begin{array}{l}2,30 \\
2,26 \\
2,28\end{array}$ & $\begin{array}{l}2,45 \\
2,39 \\
2,41\end{array}$ & $\begin{array}{l}2,65 \\
2,49 \\
2,52\end{array}$ & $\begin{array}{l}2,86 \\
2,61 \\
2,65\end{array}$ \\
\hline $\begin{array}{l}\mathrm{K} \mathbf{~ C l} . \\
\mathbf{K} \mathbf{B r} \\
\mathbf{K} \mathbf{J} .\end{array}$ & : & $\begin{array}{l}1,87 \\
2,10 \\
1,93\end{array}$ & $\begin{array}{l}1,85 \\
2,11 \\
2,07\end{array}$ & $\begin{array}{l}2,15 \\
2,21 \\
2,17\end{array}$ & $\begin{array}{l}2,26 \\
2,29 \\
2,27\end{array}$ & $\begin{array}{l}2,38 \\
2,37 \\
2,36\end{array}$ & $\begin{array}{l}2,47 \\
2,42 \\
2,45\end{array}$ & $\begin{array}{l}2,57 \\
2,50 \\
2,50\end{array}$ \\
\hline
\end{tabular}

Man erkennt aus diesen Tabellen, daß $\gamma_{0}$ and $\gamma_{\infty}$ zwar beträchtlich von $\beta$, aber sehr wenig von $n$ abhängen.

§ 6. Vergleich mit der Erfahrung. Die Elastizitätskonstanten $c_{11}$ and $c_{14}$ sind nur für 2 Kristalle, $\mathrm{NaCl}$ und $\mathrm{K} \mathrm{Cl}$, gemessen ${ }^{1}$ ), und zwar ist in CGS-Einheiten

$$
\begin{array}{cl}
\text { für } \mathrm{NaCl} & c_{11}=4,68 \cdot 10^{11} \\
n \mathrm{KCl} & c_{11}=3,68 \cdot 10^{11} .
\end{array}
$$

Wir berechnen daraus den Wert von $f_{1}$ aus der Formel (64)

$$
c_{11}=A=\frac{e^{2} S_{0}(1)}{6 r_{0}^{4}} f_{1} \text {. }
$$

Mit Benutzang der Werte (75) und (80) wird

und daraus folgt

$$
f_{1}=1,168 \cdot 10^{-13} c_{11}\left(\frac{\mu_{1}+\mu_{2}}{\rho}\right)^{3 / 4},
$$

$$
\text { für. } \begin{aligned}
\mathrm{NaCl} & f_{1}=4,42 \\
\pi \mathrm{K} \mathrm{Cl} & f_{2}=5,43 .
\end{aligned}
$$

Vergleicht man diese Werte mit denen der Tabelle 4, so findet man, daß beide sehr nahe zu der Spalte $\beta=-0,5$ gehören.

Das bedeutet nach (56), daß bezüglich der mit $r^{-n}$ proportioualen Kraft gleichartige und ungleichartige Ionen sich verschieden

1) W. Voigt, Lehrbuch der Kristallphysik, $\$ 373$, 8. 744. Eine Berechnung der ebenfalls gemessenen Konstanten $c_{12}$ liefert keine unabhängige Prüfung der Theorie, da sich $c_{12}$ durch $x$ und $c_{11}$ ausdrücken läßt $\left(\frac{3}{x}=c_{11}+2 c_{12}\right)$. 
verhalten: Ungleichartige Ionen müssen als nächste Nachbarn in Gitter sich natürlich abstoßen, weil sonst das Gitter zusammenbräche; gleichartige Ionen aber ziehen sich an. Die mit $r^{-n}$ proportionalen Strukturkräfte verhalten sich also hinsichtlich der Richtung gerade entgegengesetzt, wie die mit $r^{-1}$ proportionalen Coulombschen Kräfte. Man kann diese Anziehung gleichartiger Ionen vielleicht, damit in Zusammenhang bringen, daß ibre Elektronen nicht nur in äquivalenten Bahnen, sondern auch in gleicher Phase laufen ${ }^{1}$ ). Für die Größe dieser Kräfte findet man mit $\beta=-\frac{1}{2}$ ans (56):

$$
b_{11}^{(n)}+b_{22}^{(n)}=-b_{12}^{(n)}
$$

wenn man annimmt, daß die (auf gleiche Entfernung reduzierte) Anziehung zwischen zwei positiven Ionen etwa ebenso groß ist, wie die zwischen zwei negativen $\left(l_{11}^{(n)}=b_{22}^{(n)}\right)$, so sieht man, daß beide etwa halb so groß sind als die Abstoßung zweier verschiedener Ionen:

$$
b_{11}^{(n)}=b_{22}^{(n)}=-\frac{1}{2} b_{12}^{(n)} .
$$

Natürlich ist das nur eine rohe Schätzung.

Wir wollen nun mit diesem Werte $\beta=-0,5$ die Wellenlängen der Reststrahlen berechnen ${ }^{2}$ ).

Die Eigenfrequenz $\omega_{0}$ wird durch die Konstante $D$ bestimmt nach der Gleichung

dabei ist nach (64)

$$
\omega_{0}^{2}=\left(\frac{1}{m_{1}}+\frac{1}{m_{2}}\right) \Delta D
$$

$$
\Delta D=\frac{S_{0}(1)}{3} \frac{e^{2}}{r_{0}^{3}} f_{3} \text {. }
$$

Führt man nun die Dichte $\varrho=\frac{m_{1}+m_{2}}{2 r_{0}^{3}}$, die Faradaysche Konstante $F=N e=2,90.10^{14} \mathrm{ESE}$, die Atomgewichte $\mu_{1}=N m_{1}$. $\mu_{2}=N m_{2}$ und die Eigenwellenlänge $\lambda_{0}=\frac{2 \pi c}{\omega_{0}}$ ein, so erhält man also

$$
\omega_{*}^{2}=\frac{4 \pi^{2} c^{3}}{\lambda_{0}^{2}}=\frac{2 S_{0}(1) F^{2} \varrho}{3 \mu_{1} \mu_{2}} f_{3}
$$

$$
\lambda_{0}=\sqrt{\frac{6 \pi^{2} c^{2}}{S_{0}(1) F^{2}} \cdot \frac{\mu_{1} \mu_{2}}{\varrho f_{3}}}=6,03 \sqrt{\frac{\mu_{1} \mu_{2}}{\varrho f_{3}}} .
$$

1) M. Born, Die Naturwirsensehaften 10,677, 1922.

2) Der umgekehrte Weg, aus den Reststrahlfrequenzen die Werte von $f_{3} \mathrm{zu}$ ermitteln und mit der Tabelle 4 zu vergleichen, ist nicht vorteilhaft wegen der Unsicherheit der Daten zur Umrechnung rom Reflexionsmaximum auf die Eigenfrequenz. 
Tabelle 12.

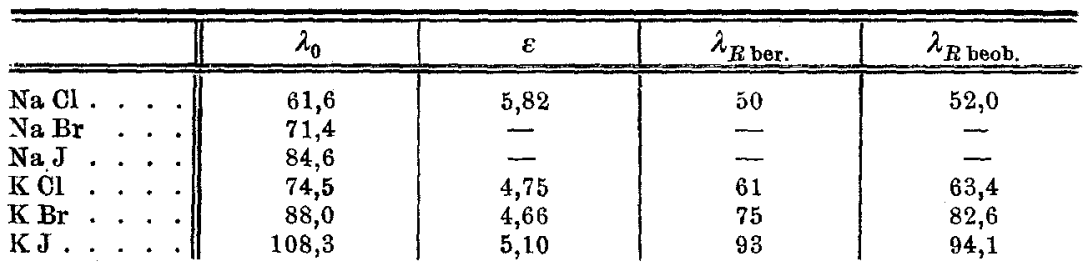

Indem man $\mu_{1}, \mu_{2}, \rho$ der Tabelle 2 und $f_{3}$ der Spalte $\beta=-0,5$ von Tabelle 6 entnimmt, erhält man die Spalte $\lambda_{0}$ der Tabelle 12. Diese Größen unterscheiden sich noch, wie Försterling ${ }^{1}$ ) bemerkt hat, beträchtlich von den beobachteten Wellenlängen der Reststrablen, die das Maximum des Reflexionsvermögens angeben. Die Korrektur gewinnt man nach Försterling folgendermaßen:

Es sei $n$ der Brechungsindex und $n_{0}$ der Anteil, der von den Elektronensehwingungen herrührt; dann gilt bei Vernachlässigung der Dämpfung für das ultrarote Gebiet außerhalb des Absorptionsstreifens selbst die Dispersionsformel ${ }^{2}$ )

$$
n^{2}=n_{0}^{2}+\frac{K}{1-\frac{\omega^{2}}{\omega_{v}^{2}}}, \quad K=\frac{4 \pi F^{2} \varrho}{\mu_{1} \mu_{2} \omega_{0}^{2}} .
$$

Die statische Dielektrizitätskonstante $\varepsilon$ ist der Wert von $n^{2}$ für $\omega=0$, also $\varepsilon=n_{0}^{2}+K$. Dann ist die Reststrahlwellenlänge

$$
\lambda_{R}=\frac{\lambda_{0}}{\sqrt{1+\frac{K}{2(\varepsilon-K)}}} .
$$

Für $K$ erhält man ans (85) und (87)

$$
K=\frac{6 \pi}{S_{0}(1)} \cdot \frac{1}{f_{3}}=\frac{10,78}{f_{3}} .
$$

In der zweiten Spalte von Tabelle 12 sind die gemessenen Werte der Dielektrizitätskonstante $\varepsilon^{3}$ ) eingetragen und damit sind die in der nächsten Spalte aufgeführten Werte von $\lambda_{R}$ berechnet. Die beobachteten Werte sind daneben gestellt. Man erkennt eine gute Ubbereinstimmung, nur bei $\mathrm{KBr}$ fällt der berechnete Wert etwas zu klein aus.

1) K. Försterling, Ann. d. Phys. (4) 61, 577, 1920.

2) M. Born, Sitzungsber. d. preuß. Akad. d. Wiss. 1918, S. 604, § 3, Formel (6), (7).

3) Diese sind aus der soeben zitierten Arbeit (Tab. 1, S. 611) entnommen, wo sie mit $D$ bezeichnet sind. 
Endlich betrachten wir die thermische Ausdebnung. Setzt man für die Atomwärme den Dulong-Petitschen Wert $C_{v}=3 R$, so erhält man für

$$
\gamma=\frac{3 V \alpha}{x C_{v}}=\frac{\mu_{1}+\mu_{2}}{2 R \varrho} \cdot \frac{\alpha}{\varkappa}
$$

die Werte ${ }^{1}$ ) der Tabelle 13 , die von den theoretischen Werten von $\gamma_{0}$ und $\gamma_{\infty}$ nach Tabelle 10 und 11, Spalte $\beta=-0,5$, umrahmt sind.

Tabelle 13.

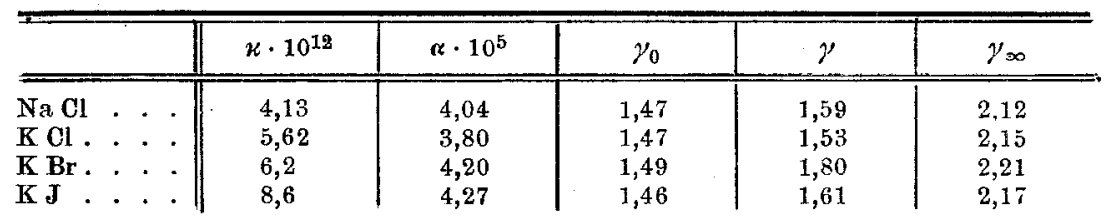

In der Tat fällt $\gamma$ zwischen $\gamma_{0}$ und $\gamma_{\infty}$, und zwar etwas näher an $\gamma_{0}$; das bedeutet, daß die ultraroten Schwingungen durchaus nicht als monochromatisch behandelt werden dürfen. Zn beachten ist anch, daß die größten Werte von $\gamma, \gamma_{0}, \gamma_{\infty}$ übereinstimmend bei $K \mathrm{Br}$ angetroffen werden; danach scheint die Hoffnung berechtigt, daß die Theorie bei weiterem Ausbau auch feinere Unterschiede der Kiristalle wiedergeben wird.

\section{Zus am menfassung.}

1. Die in einer früheren Arbeit anfgestellte Behauptung, daß das Grüneisensche Gesetz von der Proportionalität zwischen Atomwärme und thermischer Ausdehnung bei tiefen Temperaturen nicht gilt, wird als unrichtig nachgewiesen.

2. Es werden Näherungsformeln für die Temperaturabhängigkeit der Kristallkonstanten aufgestellt, die auf demselben Prinzip beruben: das Debye in die Theorie der spezifischen Wärme cingeführt hat.

3. Die Elastizitätskonstanten, die Reststiahl-Wellenlängen und der Ausdehnungskoeffizient werden für die Gitter vom Steinsalztypus durch Gittersummen ansgedrückt. Diese werden unter der Annalıme berechnet, daß die Kohäsion auf der elcktrostatischen Anziehung der Ionen beruht und außerdem eine $r^{-n}$ proportionale Kraft wirkt.

1) Dieselben Größen $\gamma$ finden sich bei Grï neisen (2. Conseil de Physique Solvay 1913 ; E. Grüneisen, Molekulartheorie der festen Körper) tabelliert, unter der Bezeichnung $\left(\frac{\partial p}{\partial \tilde{E}}\right)_{v}$, Tab. 8, S. 44. Die Abweichungen von nnseren Zahlen rähren davon her, daß Gr ̈̈n $\mathrm{e}$ isen mit individuellen $C_{v}$-Werten rechnet, während wir $C_{p}=3 R$ setzen. 
352 M. Born und E. Brody, Zur Thermodynamik der Kristallgitter II.

4. Aus den Dichten und Kompressibilitäten werden die Werte des Exponenten $n$ bestimmt. Dann ergibt sich aus dem Vergleich der berechneten Elastizitätskonstanten mit den beobachteten, daß die mit $r^{-n}$ proportionale Kraft zwischen ungleichartigen Ionen abstoßend, zwischen gleichartigen anziehend wirkt und daß die letztere, auf gleiche Entfernung reduziert, etwa halb so stark ist wie die erstere.

5. Aus denselben Daten ergeben sich die Reststrahl-Wellenlängen in guter Ubereinstimmung mit den Beobachtungen.

6. Für die von Mie und Grüneisen eingeführte Konstante $\gamma$, die im wesentlichen das Verhältnis des Ausdebnungskoeffizienten zar Atomwärme bestimmt, erhält man zwei Grenzwerte für tiefe und hohe Temperaturen. Die aus Messungen bei gewöhnlichen Temperaturen bestimmten Werte von $\gamma$ liegen zwischen diesen Grenzwerten. Daraus ist zu schließen, daß die Annahme einer monochromatischen Eigenfrequenz, die bei der Berechnung der Atomwärme neben dem kontinuierlichen Spektrum angesetzt wird, für die Darstellang der thermischen Ausdehnung nicht ausreicht. Eine genauere Theorie ist zurzeit noch nicht durchführbar. 